\title{
Uso de matérias primas da agroindústria - garapa e extrato de levedura - na produção de asparaginase por Zymomonas mobilis CP4
}

\section{Utilization of raw materials from agroindustry - sugar cane juice and yeast extract - for asparaginase production by Zymomonas mobilis CP4}

\author{
Josiani Fatima Casotti ${ }^{1}$; Doumit Camilios Neto ${ }^{1}$; Gilcelene Bruzon ${ }^{1}$; Robson Alessandro \\ Mattos Machado ${ }^{1}$; João Batista Buzato ${ }^{2}$; Maria Antonia Pedrine Colabone Celligoi ${ }^{3}$
}

\section{Resumo}

Garapa e extrato de levedura foram usados na produção de asparaginase por Zymomonas mobilis CP4. $\mathrm{Na}$ otimização utilizou metodologia de superfície de resposta com 2 variáveis (extrato de levedura e asparagina) em 3 níveis $(1,0 ; 5,5$ e 10,0 g/L) e uma repetição do ponto central. A fermentação em batelada utilizou garapa diluída a $8 \%(\mathrm{P} / \mathrm{V})$ de Açúcares Totais e inóculo de Zymomonas mobilis CP4 na concentração de $2 \mathrm{mg} / \mathrm{mL}$. Após a fermentação de 18 horas, a maior produção obtida de asparaginase foi de 9,75 U/L em extrato de levedura em 5,5 g/L e asparagina em 1,0 g/L.

Palavras-chave: Garapa, Zymomonas mobilis, asparaginase, fermentação

\begin{abstract}
Sugar cane juice and yeast extract have been used for asparaginase production by Z. mobilis CP4. A complete factorial design of two variables (yeast extract and asparagin) at three levels (1.0; 5.5 and 10.0 $\mathrm{g} / \mathrm{L}$ ) with one replication at the central point was used. Batch fermentation utilised sugar cane juice diluted at $8 \%(\mathrm{~W} / \mathrm{V})$ of Total Sugars and an inoculum of $2 \mathrm{mg}$ of cells $/ \mathrm{mL}$. After fermentation time of 18 hours, the highest production of asparaginase was $9.75 \mathrm{U} / \mathrm{L}$ using both yeast extract $(5.5 \mathrm{~g} / \mathrm{L})$ and asparagin $(1.0 \mathrm{~g} / \mathrm{l})$.
\end{abstract}

Key words: Sugar cane juice, Zymomonas mobilis, asparaginase, fermentation

\section{Introdução}

O Brasil é o maior produtor mundial de etanol a partir da fermentação da garapa e melaço de canade-açúcar. Desde o advento do PROALCOOL, o Brasil avançou na tecnologia de produção de etanol bem como no aumento da área de plantio de cana. A produção industrial de etanol gera o fermento que é comercializado para a fabricação de ração animal, suplemento alimentar humano e extrato de levedura. Ainda que a importância da produção de etanol como energia renovável para o Brasil e a geração de empregos sejam inegáveis, o açúcar da cana pode ser utilizado como substrato para a produção de metabólitos de valor agregado maior que o etanol.

\footnotetext{
1 Alunos de pós-graduação do Departamento de Bioquímica e Biotecnologia/CCE/UEL.

2 Docente do Programa de Mestrado em Biotecnologia do Departamento de Bioquímica e Biotecnologia/CCE/UEL. E-mail: buzato@uel.br.

3 Docente do Programa de Mestrado em Biotecnologia do Departamento de Bioquímica e Biotecnologia/CCE/UEL.

* Autor para correspondência
} 
A asparaginase, um agente anti-neoplásico, é utilizada no tratamento de leucemia aguda. A enzima catalisa a hidrólise de asparagina em ácido aspártico e amônia, levando a depleção de asparagina em células tumorais que são incapazes de produzir esse aminoácido. Assim a depleção de asparagina, causada pela administração endovenosa de asparaginase, provoca a morte de células malignas. $\mathrm{O}$ atual uso de asparaginase de Escherichia coli causa efeitos colaterais tóxicos em administração contínua (PRAKASHAM et al., 2007; KOTZIA et al., 2005) e além de ser de custo de produção elevado. Sendo assim, tem havido a busca de outros microrganismos produtores dessa enzima com menor toxidez bem como, capazes de usar matéria prima de baixo custo na fermentação.

Zymomonas mobilis uma bactéria gram-negativa que utiliza glicose, frutose e sacarose e produz etanol e $\mathrm{CO}_{2}$. A cepa $\mathrm{CP} 4$ foi isolada de uma bebida fermentada e consumida pelo povo de Recife, denominada de Caldo Picado (MORAIS et al., 1993). Esta bactéria vem sendo utilizada experimentalmente na fermentação da garapa de cana-de-açúcar há mais de 20 anos (VUUREN; MEYER, 1982; RHEE et al., 1984; HUERTAZ-DÍAZ; CACHO; BERNARD, 1991; TANO; BUZATO, 2003). Estudos tem demonstrado também o potencial desse microrganismo para a produção de levana (BORSANI et al., 2006), sorbitol (CAZETTA et al., 2005; VIGNOLI; CELLIGOI; SILVA, 2006), ácido glicônico (SILVEIRA et al., 1999) e mais recentemente para a produção de asparaginase (ABUD et al. 2003).

Na produção de asparaginase é importante a fonte de nitrogênio sendo prolina, uréia e sais de nitrogênio (nitrato de sódio e sulfato de amônio) não recomendadas (ABUD; PINTO; ALVES, 2005). Entretanto, o extrato de levedura fornece grande quantidade de aminoácidos (cerca de 50\% do peso), além de outros nutrientes como, vitaminas do complexo B e o ácido pantotênico enquanto que, a asparagina tem o papel de indutor na produção de asparaginase (CAMILIOS NETO, 2005). Neste contexto a garapa e o extrato de levedura, matérias primas da agroindústria de baixo custo, foram utilizados para a produção de asparaginase por Zymomonas mobilis CP4. A fermentação foi otimizada por metodologia de superfície de resposta (BOX; DRAPER, 1987) com delineamento fatorial completo $3^{2}$ das variáveis: concentração de extrato de levedura e de asparagina.

\section{Material e Métodos}

Microrganismo e Meio de preservação: Zymomonas mobilis CP4 foi preservado em Placas de Petri contendo meio sólido de composição (g/L): sacarose 20,0; extrato de levedura 2,5; $\mathrm{KH}_{2} \mathrm{PO}_{4} 1,0$; $\mathrm{MgSO}_{4} \cdot 7 \mathrm{H}_{2} \mathrm{O} 0,5 ;\left(\mathrm{NH}_{4}\right)_{2} \mathrm{SO}_{4} 1,0$ e agar 15,0. O meio foi esterilizado a $121^{\circ} \mathrm{C}$ por 20 minutos. Após incubação a $30^{\circ} \mathrm{C}$ por 24 horas. As culturas foram mantidas a $4^{\circ} \mathrm{C}$ e repicadas a cada 30 dias.

Meio de fermentação: $\mathrm{O}$ meio empregado utilizou garapa diluída a $8 \%(\mathrm{P} / \mathrm{V})$ de Açúcares Totais (AT), extrato de levedura e asparagina nas seguintes concentrações (g/L): 1,0; 5,5 e 10,0 e os sais $\mathrm{KH}_{2} \mathrm{PO}_{4}$ e $\mathrm{MgSO}_{4} \cdot 7 \mathrm{H}_{2} \mathrm{O}$ foram nas mesmas concentrações do meio de preservação. Volumes de $25 \mathrm{~mL}$ foram distribuídos em frascos Erlenmeyer de capacidade de $125 \mathrm{~mL}$ e esterilizados a $121^{\circ} \mathrm{C}$ por 20 minutos.

Planejamento fatorial para produção de asparaginase: Os experimentos foram realizados segundo os princípios da metodologia de superfície de resposta, tendo como variáveis as concentrações de extrato de levedura (E.L.) e asparagina (Asn) no meio de fermentação (Tabela 1). Foi utilizado delineamento fatorial completo $3^{2}$ com uma repetição do ponto central, totalizando 10 experimentos (Tabela 2 ). 
Tabela 1. Variáveis independentes e níveis de variação utilizados.

\begin{tabular}{cccc}
\hline \multicolumn{1}{c}{ Variáveis Originais } & \multicolumn{3}{c}{ Níveis de variação } \\
\hline & -1 & 0 & +1 \\
$\mathrm{X}_{1}-$ Concentração de extrato de levedura em $(\mathrm{g} / \mathrm{L})$ & 1,0 & 5,5 & 10,0 \\
& & & \\
$\mathrm{X}_{2}-$ Concentração de asparagina em $(\mathrm{g} / \mathrm{L})$ & 1,0 & 5,5 & 10,0 \\
\hline
\end{tabular}

Tabela 2. Delineamento fatorial completo $3^{2}$ das variáveis codificadas e decodificadas.

\begin{tabular}{ccccc}
\hline Ensaio & $\mathrm{X}_{1}$ & $\mathrm{X}_{2}$ & E.L. $(\mathrm{g} / \mathrm{L})$ & Asn. $(\mathrm{g} / \mathrm{L})$ \\
\hline 1 & -1 & -1 & 1,0 & 1,0 \\
2 & -1 & 0 & 1,0 & 5,5 \\
3 & -1 & 1 & 1,0 & 10,0 \\
4 & 0 & -1 & 5,5 & 1,0 \\
5 & 0 & 0 & 5,5 & 5,5 \\
6 & 0 & 1 & 5,5 & 10,0 \\
7 & 1 & -1 & 10,0 & 1,0 \\
8 & 1 & 0 & 10,0 & 5,5 \\
9 & 1 & 1 & 10,0 & 10,0 \\
10 & 0 & 0 & 5,5 & 5,5 \\
\hline
\end{tabular}

Fermentações em batelada: Foi usado inóculo de concentração de células de $2,0 \mathrm{mg} / \mathrm{mL}$ e em quantidade de $10 \%(\mathrm{v} / \mathrm{v})$ do volume total de fermentação. Os frascos Erlenmeyers foram incubados a $30^{\circ} \mathrm{C}$, sem agitação e sem controle de pH. Após 18 horas de incubação, a fermentação foi interrompida e a biomassa foi avaliada. O conteúdo dos frascos Erlenmeyers foi centrifugado a $9.005,8 \mathrm{~g}$, sob refrigeração $\left(4^{\circ} \mathrm{C}\right)$ por 10 minutos para separação da biomassa e sobrenadante. No sobrenadante foi avaliada a concentração de A.T. e etanol. Na biomassa foi avaliada a atividade de asparaginase.

Determinação de biomassa: A biomassa foi avaliada em leituras a $605 \mathrm{~nm}$ e comparada com uma curva de calibração. A curva de calibração foi feita da seguinte maneira. Alíquotas de suspensão de biomassa foram submetidas à secagem, até peso constante, enquanto que outra alíquota, da mesma suspensão, foi submetida em diluições para a obtenção de leituras espectrofotométricas. Em seguida os valores correspondentes ao peso seco ( $\mathrm{mg} / \mathrm{mL})$ e absorvância foram graficados.

Determinação de açúcares totais: Utilizou-se o método segundo Dubois et al. (1956). Foi usada uma curva de calibração de sacarose, variando a concentração de 0 a $100 \mu \mathrm{g} / \mathrm{mL}$. As leituras foram feitas em $490 \mathrm{~nm}$.

Determinação de etanol: Utilizou-se o método de dosagem de etanol segundo Kaye e Haag (1954). O rendimento de fermentação foi calculado segundo a equação: [(Etanol)/(0,511x Açúcar consumido)]x 100, assumindo que 1 glicose $₫ 2$ Etanol $+2 \mathrm{CO}_{2}$

Determinação de asparaginase: A atividade de asparaginase foi determinada de acordo com Imada et al. (1973). O centrifugado de células era ressuspenso em 1,0 mL de tampão tris- $\mathrm{HCl} 0,05 \mathrm{M}$, $\mathrm{pH} 8,6$ e $1,0 \mathrm{~mL}$ de asparagina $0,01 \mathrm{M}$, a $37^{\circ} \mathrm{C}$ por 30 minutos. A reação era interrompida adicionando-se 0,1 mL de ácido tricloroacético 1,5 M. A amônia liberada foi determinada pelo Método de Nessler, colocando $0,5 \mathrm{~mL}$ do clarificado centrifugado da reação em 7,0 mL de água bidestilada e $1,0 \mathrm{~mL}$ do Reagente de Nessler a temperatura ambiente por 10 minutos, realizando leitura em $480 \mathrm{~nm}$. O branco usado foi uma amostra com tempo de reação de zero minuto. A concentração de amônia foi determinada com base em uma curva de calibração construída 
com solução de sulfato de amônio. Uma unidade de asparaginase (U) foi a quantidade de enzima necessária para liberar $1 \mu \mathrm{Mol}$ de amônia por minuto.

\section{Resultados e Discussão}

Zymomonas mobilis foi cultivada em garapa diluída na concentração de açúcares totais de 78,48 g/L. O consumo de açúcar variou de 84 a $90 \%$ e a produção de etanol atingiu valores teóricos de 66,3 a $98,8 \%$. Os valores elevados de consumo de açúcar e produção de etanol indicam que a fermentação foi completa, com alta atividade metabólica do microrganismo nas condições testadas.

A asparaginase é obtida industrialmente pelo cultivo de Escherichia coli. Entretanto, outros microrganismos também têm sido considerados como potenciais produtores desta enzima. Recentemente Zymomonas mobilis vem sendo estudada em fermentações objetivando a produção de asparaginase. Galani, Drainas e Typas (1985) foram os pioneiros em relatar a presença de asparaginase em fermentações conduzidas por Zymomonas mobilis. Esses autores relataram que a maior atividade de asparaginase foi obtida quando o meio de cultura continha asparagina. O interesse da asparaginase de Zymomonas mobilis foi retomado mais recentemente quando Abud et al. (2003) relataram estudos de produção em meio de glicose e asparagina. A atividade enzimática obtida foi de 4,6 U asparaginase/g células.

Camilios Neto (2005) e Camilios Neto, Buzato e Borsato (2006) otimizaram as condições de cultivo utilizando delineamento fatorial na produção de asparaginase por Zymomonas mobilis. Foi usado melaço diluído a $10 \%$ de Açúcares Redutores Totais acrescido de extrato de levedura e asparagina. Os resultados obtidos indicaram que o extrato de levedura mesmo na concentração mínima de 2,0 g/L estava em excesso. Entretanto a adição de asparagina ao meio de fermentação aumentou a atividade de asparaginase. $\mathrm{O}$ maior valor obtido de asparaginase foi de 19,90 U/L quando se usou extrato de levedura em 2,0 g/L, na presença de asparagina (variável discreta) e em 21 horas de fermentação.

Este trabalho avaliou produção de asparaginase na fermentação da garapa, no qual foi otimizado, o efeito do extrato de levedura e de asparagina através de metodologia de superfície de resposta. Os resultados obtidos são apresentados na Tabela 3 e Figura 1.

Tabela 3. Valores finais de biomassa, açúcar consumido, rendimento de fermentação e atividade de asparaginase na fermentação da garapa por Zymomonas mobilis CP4.

\begin{tabular}{ccccc}
\hline Ensaio & $\begin{array}{c}\text { Biomassa } \\
(\mathrm{g} / \mathrm{L})\end{array}$ & $\begin{array}{c}\text { Açúcar consumido } \\
(\%)\end{array}$ & $\begin{array}{c}\text { Rendimento de } \\
\text { fermentação(\%) }\end{array}$ & asparaginase (U/L) \\
\hline 1 & 0,91 & 86 & 70,45 & 5,75 \\
2 & 0,87 & 89 & 95,69 & 5,75 \\
3 & 0,79 & 84 & 86,56 & 6,00 \\
4 & 1,00 & 88 & 99,20 & 9,75 \\
5 & 0,98 & 90 & 76,40 & 7,25 \\
6 & 0,93 & 90 & 66,30 & 7,25 \\
7 & 0,94 & 89 & 98,80 & 5,50 \\
8 & 0,72 & 90 & 90,00 & 5,75 \\
9 & 0,74 & 87 & 83,40 & 2,00 \\
10 & 0,98 & 86 & 98,10 & 7,75 \\
\hline
\end{tabular}

Os valores apresentados na Tabela 1 foram analisados pela a metodologia de superfície de resposta (programa computacional STATÍSTICA 7.1) e obteve-se a seguinte equação: $\mathrm{Y}=3,90817+$
$1,7948 X_{1}+0,24537 X_{2}-0,1543 X_{1}^{2}-0,0185 X_{2}^{2}-$ $0,0463 \mathrm{X}_{1} \mathrm{X}_{2}$; onde: $\mathrm{Y}=$ atividade de asparaginase; $\mathrm{X}_{1}=$ concentração de extrato de levedura; $\mathrm{X}_{2}=$ concentração de asparagina. A análise estatística 
mostrou que a variável $X_{1}$ como a interação $X_{1}$ e $X_{2}$ foram significativas e a variável asparagina $\left(X_{2}\right)$ não foi significativa $(p<0,5)$. A otimização da equação preditiva utilizando o aplicativo OPGRAD, mostrou que a melhor condição para produção de asparaginase foi a que utilizou concentração de extrato de levedura $5,5 \mathrm{~g} / \mathrm{L}$ e concentração de asparagina de $1,0 \mathrm{~g} / \mathrm{L}$, obtendo-se uma produção de 9,75 U/L. A figura de superfície de resposta (Figura 1) ilustra o efeito do extrato de levedura e da asparagina no meio de cultivo para a produção de asparaginase.

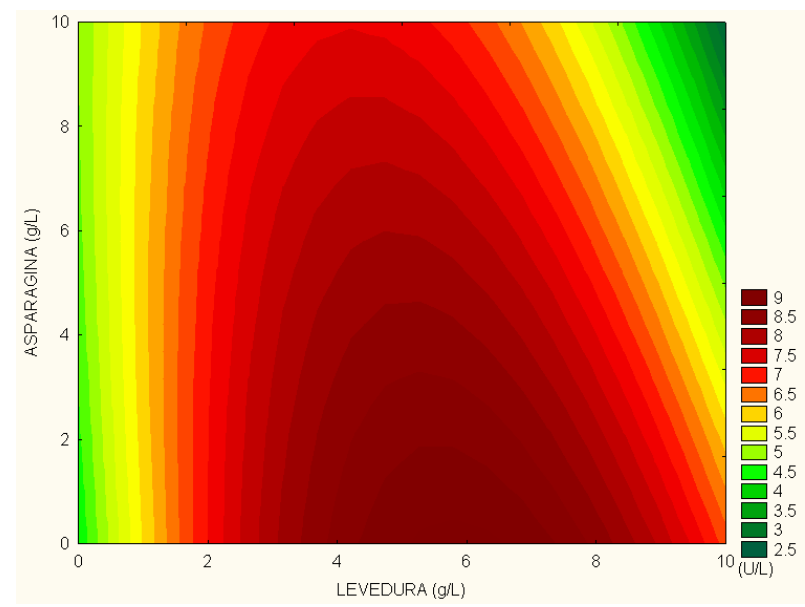

Figura 1. Superfície de resposta para a produção de asparaginase por Zymomonas mobilis $\mathrm{CP} 4$

Embora a garapa seja um substrato rico e constitui excelente meio para crescimento de microrganismos, Camilios Neto (2005) e Camilios Neto, Buzato e Borsato (2006) obtiveram valores mais elevados de asparaginase na fermentação do melaço. Considerando que a composição do melaço seja pobre em vitaminas e outros fatores de crescimento, e além de apresentar fatores de inibição como sais em excesso (principalmente de potássio e cloreto), o melaço estimularia a expressão enzimática de microrganismo melhor adaptado, explicando os nossos resultados.

\section{Conclusão}

Entre as condições testadas, a melhor produção de asparaginase de 9,75 U/L, ocorreu quando em presença de extrato de levedura a $5,5 \mathrm{~g} / \mathrm{L}$ e de asparagina a 1,0 g/L. A variação de extrato de levedura foi significativa para produção de asparaginase por Zymomonas mobilis CP4 na fermentação de garapa.

\section{Agradecimentos}

Doumit Camilios Neto, Gilcelene Bruzon e Robson Alessandro Mattos Machado agradecem o apoio financeiro cedido pelo Conselho Nacional de Desenvolvimento Científico e Tecnológico (CNPq) e Fundação Araucária.

\section{Referências}

ABUD, A. K. S.; CARNEIRO, C.C.; ALVES, T.L. M.; PINTO, J. C.; PINHEIRO, I. O. Estudo da cinética da produção de asparaginase por Zymomonas mobilis. In: SIMPÓSIO NACIONAL DE FERMENTACÕES, 14., 2003, Florianópolis. Anais... Florianópolis: UFSC, 2003. CD-ROM.

ABUD, A. K. S.; PINTO, J. C.; ALVES, T. L. Influência das fontes de carbono e nitrogênio na produção da enzima asparaginase por Zymomonas mobilis. In: SIMPÓSIO NACIONAL DE BIOPROCESSOS, 15., 2005, Recife.Anais... Recife: UFPe, 2005. CD-ROM.

BORSANI, R. R. J.; CELLIGOI, M. A. P. C.; BUZATO, J. B.; SILVA, R. S. F. Influence of carbon source and fermentation process on levan production by Zymomonas mobilis analyzed by surface response method. Ciência e Tecnologia de Alimentos, Campinas, v.26, n.3, p.604-609, 2006.

BOX, G. E. P.; DRAPER, N. Empirical model-building and response surfaces. New York: John Wiley \& Sons, 1987.

CAMILIOS NETO, D. L-asparaginase de Zymomonas mobilis na fermentação de melaço: otimização das condições de cultivo utilizando delineamento fatorial. 2005. Dissertação (Mestrado em Biotecnologia) - Universidade Estadual de Londrina, Londrina.

CAMILIOS NETO, D.; BUZATO, J. B.; BORSATO, D. Lasparaginase production by Zymomonas mobilis during molasses fermentation: optimazation of culture conditions using fatorial design. Acta Scientiarum: Technology, Maringá, v.28, n.2, p.151-153, 2006.

CAZETTA, M. L.; CELLIGOI, M. A. P. C.; BUZATO, J. B.; SCARMINO, I. S.; SILVA, R. S. F. Optimization study for sorbitol production by Zymomonas mobilis in sugar cane molasses. Process Biochemistry, London, v.40, n.2, p.747$751,2005$. 
DUBOIS, M.; GILLES, K. A.; HAMILTON, J. K.; REBERS, P. A.; SMITH, F. Colorimetric method for determination of sugard and related substances. Anaytical Chemistry, Washington, v.28, n.3, p.350-356, 1956.

GALANI, I.; DRAINAS, C.; TYPAS, M. A. Growth requirements and establishment of a chemically defined minimal medium in Zymomonas mobilis. Biotechnology Letters, Dordrecht, v.7, p.673-679, 1985.

HUERTAZ-DÍAZ, H.; CACHO, C. L.; BERNARD, L. Fermentation of sugarcane juice and blackstrap molasses by Zymomonas mobilis. Journal of Agriculture of the University of Puerto Rico, Rio Piedras, v.75, n.1, p.43-50, 1991.

IMADA, A.; IGARASI, S.; NAKAHAMA, K.; ISONO, M. Asparaginase and glutaminase activities of microorganisms. Journal of General Microbiology, Great Britain, v.76, n.1, p.85-99, 1973.

KAYE, S.; HAAG, H. B. Determination of alcohol content. Journal Forensic Medicine, Cape Town, v.4, n.1, p.373, 1954.

MORAIS, J. O. F.; RIOS, E. M. M. M.; CALAZANS, G. M. T.; LOPES, C. E. Zymomonas mobilis research in the Pernambuco Federal University. Journal of Biotechnology, Amsterdam, v.31, n.1, p.75-91, 1993.
PRAKASHAM, R. S.; RAO, C. S.; RAO, R. S.; LAKSHMI, G. S.; SARMA, P. N. L-Asparaginase production by isolated Staphyloccus sp. Journal of Applied Microbiology, Oxford, v.102, n. 5, p.1382-1391, 2007

RHEE, S. K.; PAGAN, R. J.; LEFEBVRE, M.; WONG, L.; ROGERS, P. L. Ethanol production from desalted molasses using Saccharomyces uvarum and Zymomonas mobilis. Journal of Fermentation Technology, Osaka, v.62, n.3, p.297-300, 1984.

SILVEIRA, M. M.; WISBERCK, E.; LEMMEL, C.; ERZINGER, G.; COSTA, J. P. L; BERTASSO, M. Bioconversion of glucose and fructose to sorbitol and gluconic acid by untreated cells of Zymomonas mobilis. Journal of Biotechnology, Amsterdam, n.75, p.99-103, 1999.

TANO, M. S.; BUZATO, J. B. Effect of the presence of initial ethanol on ethanol production in sugar cane juice fermented by Zymomonas mobilis. Brazilian Journal of Microbiology, São Paulo, v.34, n.3, p.242-244, 2003.

VIGNOLI, J. A.; CELLIGOI, M. A. P. C.; SILVA, R. S. F. Development of a statistical model for sorbitol production by free and immobilized Zymomonas mobilis in loofa sponge Luffa cylindrica. Process Biochemistry, London, v.41, n.1, p.240-243, 2006.

VUUREN, H. J. J.; MEYER, L. Production of ethanol from sugar cane molasses by Zymomonas mobilis. Biotechnology Letters, Dordrecht, v.4, n.4, p.253-256, 1982. 